\title{
Iterates of Bernstein Type Operators on a Triangle with All Curved Sides
}

\author{
Teodora Cătinaş \\ Faculty of Mathematics and Computer Science, Babeş-Bolyai University, 1 Kogălniceanu Cluj-Napoca, Romania \\ Correspondence should be addressed to Teodora Cătinaş; tcatinas@math.ubbcluj.ro
}

Received 2 December 2013; Accepted 17 January 2014; Published 6 March 2014

Academic Editor: Naseer Shahzad

Copyright (C) 2014 Teodora Cătinaş. This is an open access article distributed under the Creative Commons Attribution License, which permits unrestricted use, distribution, and reproduction in any medium, provided the original work is properly cited.

We consider some Bernstein-type operators as well as their product and Boolean sum for a function defined on a triangle with all curved sides. Using the weakly Picard operators technique and the contraction principle, we study the convergence of the iterates of these operators.

\section{Bernstein Type Operators}

In this paper, using the weakly Picard operators technique and the contraction principle, we study the convergence of the iterates of some operators introduced in [1]. Similar operators with the ones from [1] were studied in $[2,3]$ and [4], where the authors construct interpolation and Bernsteintype operators on triangles and squares with one and all curved sides. They studied the operators, their product and Boolean sum, as well as their interpolation properties, the order of accuracy, and the remainder of the corresponding approximation formulas.

We recall some results regarding Bernstein-type operators on a triangle with all curved sides from [1].

We denote by $\widetilde{T}_{h}$ the triangle with all curved sides, which has the vertices $V_{1}=(0, h), V_{2}=(h, 0), V_{3}=(0,0)$, and the three curved sides $\gamma_{1}, \gamma_{2}$ (along the coordinate axis), and $\gamma_{3}$ (opposite to the vertex $V_{3}$ ); $h \in \mathbb{R}_{+}$. We have that $\gamma_{1}$ is defined by $\left(x, f_{1}(x)\right)$, with $f_{1}(0)=f_{1}(h)=0, f_{1}(x) \leq 0$, for $x \in[0, h]$; $\gamma_{2}$ is defined by $\left(g_{2}(y), y\right)$, with $g_{2}(0)=g_{2}(h)=0, g_{2}(y) \leq 0$, for $y \in[0, h]$ and $\gamma_{3}$ is defined by the one-to-one functions $f_{3}$ and $g_{3}$, where $g_{3}$ is the inverse of the function $f_{3}$; that is, $y=$ $f_{3}(x)$ and $x=g_{3}(y)$, with $x, y \in[0, h]$ and $f_{3}(0)=g_{3}(0)=h$ (see Figure 1). In the sequel we denote by $e_{i j}(x, y)=x^{i} y^{j}$, for $i, j \in \mathbb{N}$.

Let $F$ be a real-valued function defined on $\widetilde{T}_{h}$ and $\left(g_{2}(y), y\right),\left(g_{3}(y), y\right)$, respectively, and let $\left(x, f_{1}(x)\right),\left(x, f_{3}(x)\right)$ be the points in which the parallel lines to the coordinate axes, passing through the point $(x, y) \in \widetilde{T}_{h}$, intersecting the sides $\gamma_{1}, \gamma_{2}$, and $\gamma_{3}$. We consider the uniform partitions of the intervals $\left[g_{2}(y), g_{3}(y)\right]$ and $\left[f_{1}(x), f_{3}(x)\right], x, y \in[0, h]:$

$$
\Delta_{m}^{x}=\left\{g_{2}(y)+i \frac{g_{3}(y)-g_{2}(y)}{m} \mid i=\overline{0, m}\right\},
$$

respectively,

$$
\Delta_{n}^{y}=\left\{f_{1}(x)+j \frac{f_{3}(x)-f_{1}(x)}{n} \mid j=\overline{0, n}\right\},
$$

and the Bernstein-type operators $B_{m}^{x}$ and $B_{n}^{y}$ defined by

$$
\begin{aligned}
& \left(B_{m}^{x} F\right)(x, y) \\
& \quad=\sum_{i=0}^{m} p_{m, i}(x, y) F\left(g_{2}(y)+i \frac{g_{3}(y)-g_{2}(y)}{m}, y\right),
\end{aligned}
$$

with

$$
\begin{aligned}
& p_{m, i}(x, y) \\
& \quad=\left(\begin{array}{c}
m \\
i
\end{array}\right)\left[\frac{x-g_{2}(y)}{g_{3}(y)-g_{2}(y)}\right]^{i}\left[1-\frac{x-g_{2}(y)}{g_{3}(y)-g_{2}(y)}\right]^{m-i},
\end{aligned}
$$




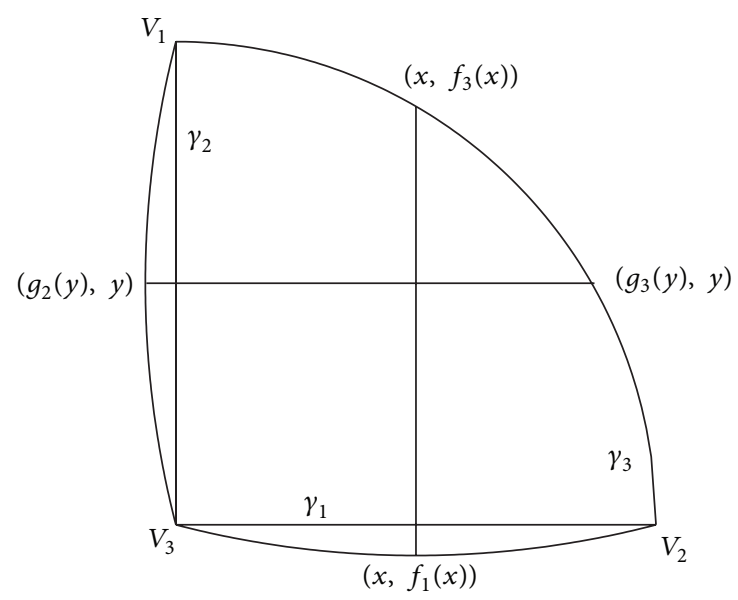

Figure 1: Triangle $\widetilde{T}_{h}$.

respectively,

$$
\begin{aligned}
& \left(B_{n}^{y} F\right)(x, y) \\
& \quad=\sum_{j=0}^{n} q_{n, j}(x, y) F\left(x, f_{1}(x)+j \frac{f_{3}(x)-f_{1}(x)}{n}\right),
\end{aligned}
$$

with

$$
q_{n, j}(x, y)=\left(\begin{array}{l}
n \\
j
\end{array}\right)\left[\frac{y-f_{1}(x)}{f_{3}(x)-f_{1}(x)}\right]^{j}\left[1-\frac{y-f_{1}(x)}{f_{3}(x)-f_{1}(x)}\right]^{n-j}
$$

Remark 1. In Figures 2(a) and 2(b) we plot the points $\left(g_{2}(y)+i\left(\left(g_{3}(y)-g_{2}(y)\right) / m\right), y\right), i=\overline{0, m}$ and, respectively, $\left(x, f_{1}(x)+j\left(\left(f_{3}(x)-f_{1}(x)\right) / n\right), j=\overline{0, n}\right.$, for $x, y \in[0, h]$.

Theorem 2. If $F$ is a real-valued function defined on $\widetilde{T}_{h}$, then

(i) $B_{m}^{x} F=F$ on $\gamma_{2} \cup \gamma_{3}$,

(ii) $B_{n}^{y} F=F$ on $\gamma_{1} \cup \gamma_{3}$,

(iii) $\left(B_{m}^{x} e_{i 0}\right)(x, y)=x^{i}, i=0,1$,

$$
\begin{aligned}
& \left(B_{m}^{x} e_{20}\right)(x, y)=x^{2}+\left[x-g_{2}(y)\right]\left[g_{3}(y)-x\right] / m, \\
& \left(B_{m}^{x} e_{i j}\right)(x, y)=y^{j}\left(B_{m}^{x} e_{i 0}\right)(x, y), i=0,1,2 ; j \in \mathbb{N},
\end{aligned}
$$

(iv) $\left(B_{n}^{y} e_{0 j}\right)(x, y)=y^{j}, j=0,1$,

$$
\begin{aligned}
& \left(B_{n}^{y} e_{02}\right)(x, y)=y^{2}+\left[y-f_{1}(x)\right]\left[f_{3}(x)-y\right] / n, \\
& \left(B_{n}^{y} e_{i j}\right)(x, y)=x^{i}\left(B_{n}^{y} e_{0 j}\right)(x, y), j=0,1,2 ; i \in \mathbb{N} .
\end{aligned}
$$

Let $P_{m n}=B_{m}^{x} B_{n}^{y}$, respectively, and let $Q_{n m}=B_{n}^{y} B_{m}^{x}$ be the products of the operators $B_{m}^{x}$ and $B_{n}^{y}$.
We have

$$
\begin{aligned}
& \left(P_{m n} F\right)(x, y) \\
& =\sum_{i=0}^{m} \sum_{j=0}^{n} p_{m, i}(x, y) q_{n, j}\left(x_{i}, y\right) \\
& \quad \times F\left(x_{i}, f_{1}\left(x_{i}\right)+j \frac{f_{3}\left(x_{i}\right)-f_{1}\left(x_{i}\right)}{n}\right)
\end{aligned}
$$

with $x_{i}=g_{2}(y)+i\left(\left(g_{3}(y)-g_{2}(y)\right) / m\right)$, respectively,

$$
\begin{aligned}
& \left(Q_{n m} F\right)(x, y) \\
& =\sum_{i=0}^{m} \sum_{j=0}^{n} p_{m, i}\left(x, y_{j}\right) q_{n, j}(x, y) \\
& \quad \times F\left(g_{2}\left(y_{j}\right)+i \frac{g_{3}\left(y_{j}\right)-g_{2}\left(y_{j}\right)}{m}, y_{j}\right),
\end{aligned}
$$

with $y_{j}=f_{1}(x)+j\left(\left(f_{3}(x)-f_{1}(x)\right) / n\right)$.

Theorem 3. If $F$ is a real-valued function defined on $\widetilde{T}_{h}$, then

(i) $\left(P_{m n} F\right)\left(V_{3}\right)=F\left(V_{3}\right), P_{m n} F=F$, on $\gamma_{3}$ and

(ii) $\left(Q_{n m} F\right)\left(V_{3}\right)=F\left(V_{3}\right), Q_{n m} F=F$, on $\gamma_{3}$.

We consider the Boolean sums of the operators $B_{m}^{x}$ and $B_{n}^{y}$; that is,

$$
S_{m n}:=B_{m}^{x} \oplus B_{n}^{y}=B_{m}^{x}+B_{n}^{y}-B_{m}^{x} B_{n}^{y},
$$

respectively,

$$
T_{n m}:=B_{n}^{y} \oplus B_{m}^{x}=B_{n}^{y}+B_{m}^{x}-B_{n}^{y} B_{m}^{x} .
$$

Theorem 4. If $F$ is a real-valued function defined on $\widetilde{T}_{h}$, then

$$
\begin{gathered}
\left.S_{m n} F\right|_{\partial \widetilde{T}_{h}}=\left.F\right|_{\partial \widetilde{T}_{h}}, \\
\left.T_{n m} F\right|_{\partial \widetilde{T}_{h}}=\left.F\right|_{\partial \widetilde{T}_{h}} .
\end{gathered}
$$

\section{Weakly Picard Operators}

We recall some results regarding weakly Picard operators that will be used in the sequel (see, e.g., [5]).

Let $(X, d)$ be a metric space and $A: X \rightarrow X$ an operator. We denote by $F_{A}:=\{x \in X \mid A(x)=x\}$, the fixed points set of $A ; I(A):=\{Y \subset X \mid A(Y) \subset Y, Y \neq \emptyset\}$, the family of the nonempty invariant subsets of $A ; A^{0}:=1_{X}, A^{1}:=$ $A, \ldots, A^{n+1}:=A \circ A^{n}, n \in \mathbb{N}$.

Definition 5. The operator $A: X \rightarrow X$ is a Picard operator if there exists $x^{*} \in X$ such that

(i) $F_{A}=\left\{x^{*}\right\}$;

(ii) the sequence $\left(A^{n}\left(x_{0}\right)\right)_{n \in \mathbb{N}}$ converges to $x^{*}$ for all $x_{0} \in$ $X$. 


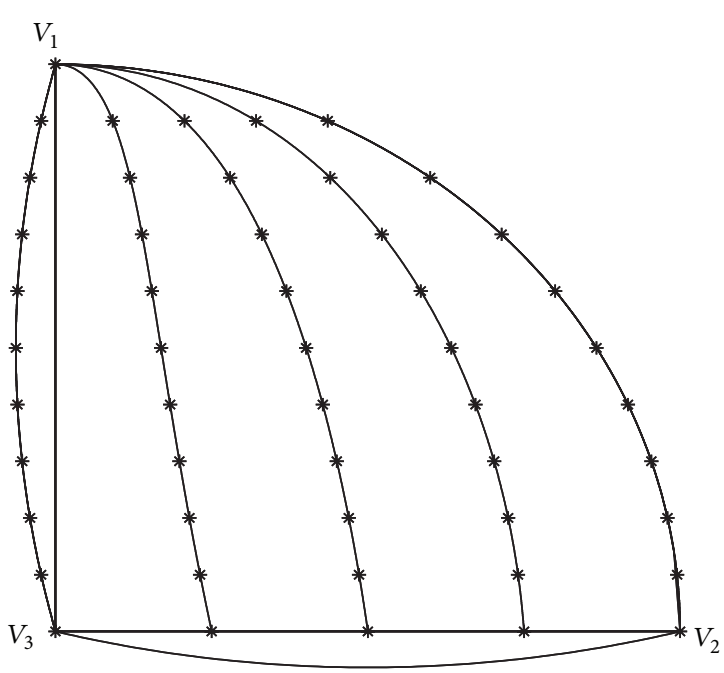

(a)

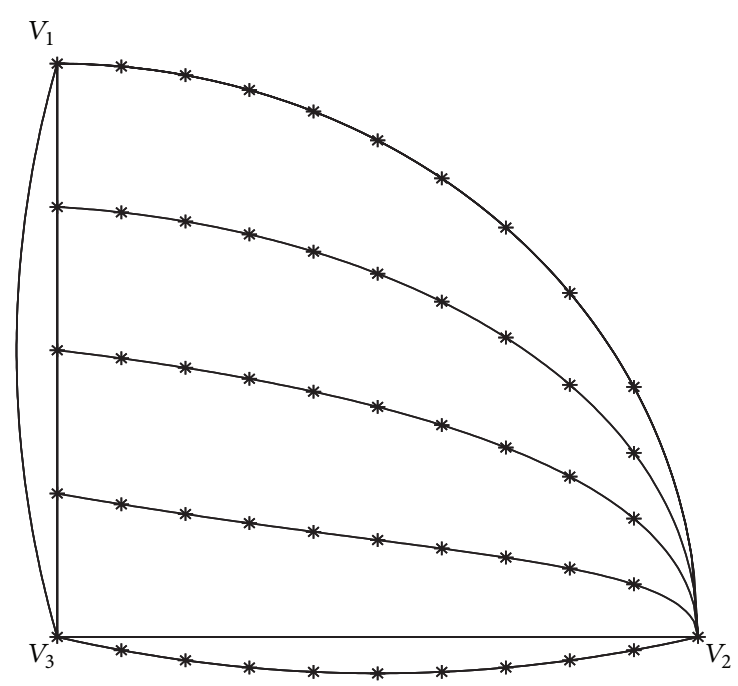

(b)

Figure 2: (a) Points of $\Delta_{m}^{x}$, for $m=4$. (b) Points of $\Delta_{y}^{n}$, for $n=4$.

Definition 6. The operator $A$ is a weakly Picard operator if the sequence $\left(A^{n}(x)\right)_{n \in \mathbb{N}}$ converges, for all $x \in X$, and the limit (which may depend on $x$ ) are a fixed point of $A$.

Definition 7. If $A$ is a weakly Picard operator, then we consider the operator $A^{\infty}, A^{\infty}: X \rightarrow X$, defined by

$$
A^{\infty}(x):=\lim _{n \rightarrow \infty} A^{n}(x) .
$$

Theorem 8. An operator $A$ is a weakly Picard operator if and only if there exists a partition of $X, X=\bigcup_{\lambda \in \Lambda} X_{\lambda}$, such that

(a) $X_{\lambda} \in I(A), \forall \lambda \in \Lambda$;

(b) $\left.A\right|_{X_{\lambda}}: X_{\lambda} \rightarrow X_{\lambda}$ is a Picard operator, $\forall \lambda \in \Lambda$.

\section{Iterates of Bernstein Type Operators}

Let $F$ be a real-valued function defined on $\widetilde{T}_{h} ; h \in \mathbb{R}_{+}$.

Using the weakly Picard operators technique and the contraction principle, we study the convergence of the iterates of the Bernstein-type operators (3) and (5) and of their product and Boolean sum operators (7), (8), (9) and (10). The same approach for some other linear and positive operators leads to similar results in [6-12].

The limit behavior for the iterates of some classes of positive linear operators was also studied, for example, in [1323]. In the papers [19-21] new methods were introduced (e.g., Korovkin type technique) for the study of the asymptotic behavior of the iterates of positive linear operators, positive linear operators preserving the affine functions and defined on the space of bounded real-valued functions on $[0,1]$. This techniques enlarge the class of operators for which the limit of the iterates can be computed. In $[13,14]$ some methods were proposed to determine the degree of convergence for the iterates of certain positive linear operators towards the first Bernstein operator. Using the spectrum of the operators involved in [15], convergence results were proved for overiterates of certain (generalized) Bernstein-Stancu operators (see, e.g., [24-26]). In [16, 17] new techniques were introduced (infinite products, rates of convergence), based on the results from [18], in order to prove that infinite products of certain positive linear operators weakly converge to the first Bernstein operator.

Now we study the convergence of the iterates of the Bernstein-type operators (3) and (5).

Theorem 9. The operators $B_{m}^{x}$ and $B_{n}^{y}$ are weakly Picard operators and

$$
\begin{aligned}
\left(B_{m}^{x, \infty} F\right)(x, y) & \\
= & \frac{F\left(g_{3}(y), y\right)-F\left(g_{2}(y), y\right)}{g_{3}(y)-g_{2}(y)} x \\
& +\frac{g_{3}(y) F\left(g_{2}(y), y\right)-g_{2}(y) F\left(g_{3}(y), y\right)}{g_{3}(y)-g_{2}(y)}, \\
\left(B_{n}^{y, \infty} F\right)(x, y) & \\
= & \frac{F\left(x, f_{3}(x)\right)-F\left(x, f_{1}(x)\right)}{f_{3}(x)-f_{1}(x)} y \\
& +\frac{f_{3}(x) F\left(x, f_{1}(x)\right)-f_{1}(x) F\left(x, f_{3}(x)\right)}{f_{3}(x)-f_{1}(x)} .
\end{aligned}
$$

Proof. Taking into account the interpolation properties of $B_{m}^{x}$ and $B_{n}^{y}$ (from Theorem 2), let us consider

$$
\begin{aligned}
X_{\left.\varphi\right|_{\gamma_{2}},\left.\varphi\right|_{\gamma_{3}}}^{(1)}=\left\{F \in C\left(\widetilde{T}_{h}\right)\left|F\left(g_{2}(y), y\right)=\varphi\right|_{\gamma_{2}},\right. \\
\\
\left.F\left(g_{3}(y), y\right)=\left.\varphi\right|_{\gamma_{3}}\right\}, \text { for } y \in[0, h],
\end{aligned}
$$




$$
\begin{aligned}
X_{\left.\psi\right|_{1}}^{(2)},\left.\psi\right|_{\gamma_{3}}=\left\{F \in C\left(\widetilde{T}_{h}\right)\left|F\left(x, f_{1}(x)\right)=\psi\right|_{\gamma_{1}},\right. \\
\\
\left.F\left(x, f_{3}(x)\right)=\left.\psi\right|_{\gamma_{3}}\right\}, \quad \text { for } x \in[0, h]
\end{aligned}
$$

and denote

$$
\begin{aligned}
F_{\left.\varphi\right|_{\gamma_{2}},\left.\varphi\right|_{\gamma_{3}} ^{(1)}(x, y):=} & \frac{\left.\varphi\right|_{\gamma_{3}}-\left.\varphi\right|_{\gamma_{2}}}{g_{3}(y)-g_{2}(y)} x \\
& +\frac{\left.g_{3}(y) \varphi\right|_{\gamma_{2}}-\left.g_{2}(y) \varphi\right|_{\gamma_{3}}}{g_{3}(y)-g_{2}(y)}, \\
F_{\left.\psi\right|_{\gamma_{1}},\left.\psi\right|_{\gamma_{3}}}^{(2)}(x, y):= & \frac{\left.\psi\right|_{\gamma_{3}}-\left.\psi\right|_{\gamma_{1}}}{f_{3}(x)-f_{1}(x)} y \\
& +\frac{\left.f_{3}(x) \psi\right|_{\gamma_{1}}-\left.f_{1}(x) \psi\right|_{\gamma_{3}}}{f_{3}(x)-f_{1}(x)},
\end{aligned}
$$

with $\varphi, \psi \in C\left(\widetilde{T}_{h}\right)$.

We have the following properties:

(i) $X_{\left.\varphi\right|_{\gamma_{2}},\left.\varphi\right|_{\gamma_{3}}}^{(1)}$ and $X_{\left.\psi\right|_{\gamma_{1}},\left.\psi\right|_{\gamma_{3}}}^{(2)}$ are closed subsets of $C\left(\widetilde{T}_{h}\right)$;

(ii) $X_{\left.\varphi\right|_{\gamma_{2}},\left.\varphi\right|_{\gamma_{3}}}^{(1)}$ is an invariant subset of $B_{m}^{x}$ and $X_{\left.\psi\right|_{\gamma_{1}},\left.\psi\right|_{\gamma_{3}}}^{(2)}$ is an invariant subset of $B_{n}^{y}$, for $\varphi, \psi \in C\left(\widetilde{T}_{h}\right)$ and $n, m \in$ $\mathbb{N}^{*}$;

(iii) $C\left(\widetilde{T}_{h}\right)=\bigcup_{\varphi \in C\left(\widetilde{T}_{h}\right)} X_{\left.\varphi\right|_{\gamma_{2}},\left.\varphi\right|_{\gamma_{3}}}^{(1)}$ and $C\left(\widetilde{T}_{h}\right)=$ $\bigcup_{\psi \in C\left(\widetilde{T}_{h}\right)} X_{\left.\psi\right|_{\gamma_{1}},\left.\psi\right|_{\gamma_{3}}}^{(2)}$ are partitions of $C\left(\widetilde{T}_{h}\right)$;

(iv) $F_{\left.\varphi\right|_{\gamma_{2}},\left.\varphi\right|_{\gamma_{3}}}^{(1)} \in X_{\left.\varphi\right|_{\gamma_{2}},\left.\varphi\right|_{\gamma_{3}}}^{(1)} \cap F_{B_{m}^{x}}$ and $F_{\left.\psi\right|_{\gamma_{1}},\left.\psi\right|_{\gamma_{3}}}^{(2)} \in X_{\left.\psi\right|_{\gamma_{1}},\left.\psi\right|_{\gamma_{3}}}^{(2)} \cap$ $F_{B_{n}^{y}}$, where $F_{B_{m}^{x}}$ and $F_{B_{n}^{y}}$ denote the fixed points sets of $B_{m}^{x}$ and $B_{n}^{y}$.

The statements (i) and (iii) are obvious.

(ii), by linearity of Bernstein operators and Theorem 2, it follows that $\forall F_{\left.\varphi\right|_{\gamma_{2}},\left.\varphi\right|_{\gamma_{3}}}^{(1)} \in X_{\left.\varphi\right|_{\gamma_{2}},\left.\varphi\right|_{\gamma_{3}}}^{(1)}$ and $\forall F_{\left.\psi\right|_{\gamma_{1}},\left.\psi\right|_{\gamma_{3}}}^{(2)} \in X_{\left.\psi\right|_{\gamma_{1}},\left.\psi\right|_{\gamma_{3}}}^{(2)}$; we have

$$
\begin{aligned}
& B_{m}^{x} F_{\left.\varphi\right|_{\gamma_{2}},\left.\varphi\right|_{\gamma_{3}}}^{(1)}(x, y)=F_{\left.\varphi\right|_{\gamma_{2}},\left.\varphi\right|_{\gamma_{3}}}^{(1)}(x, y), \\
& B_{n}^{y} F_{\left.\psi\right|_{\gamma_{1}},\left.\psi\right|_{\gamma_{3}}}^{(2)}(x, y)=F_{\left.\psi\right|_{\gamma_{1}},\left.\psi\right|_{\gamma_{3}}}^{(2)}(x, y) .
\end{aligned}
$$

So, $X_{\left.\varphi\right|_{\gamma_{2}},\left.\varphi\right|_{\gamma_{3}}}^{(1)}$ and $X_{\left.\psi\right|_{\gamma_{1}},\left.\psi\right|_{\gamma_{3}}}^{(2)}$ are invariant subsets of $B_{m}^{x}$ and, respectively, of $B_{n}^{y}$, for $\varphi, \psi \in C\left(\widetilde{T}_{h}\right)$ and $n, m \in \mathbb{N}^{*}$.

(iv), we prove that

$$
\begin{aligned}
& \left.B_{m}^{x}\right|_{X_{\left.\varphi\right|_{\gamma_{2}},\left.\varphi\right|_{\gamma_{3}}}^{(1)}}: X_{\left.\varphi\right|_{\gamma_{2}},\left.\varphi\right|_{\gamma_{3}} ^{(1)}}^{(1)} \longrightarrow X_{\left.\varphi\right|_{\gamma_{2}},\left.\varphi\right|_{\gamma_{3}} ^{(1)}}, \\
& \left.B_{n}^{y}\right|_{X_{\left.\psi\right|_{\gamma_{1}},\left.\psi\right|_{\gamma_{3}}}^{(2)}}: X_{\left.\psi\right|_{\gamma_{1}},\left.\psi\right|_{\gamma_{3}}}^{(2)} \longrightarrow X_{\left.\psi\right|_{\gamma_{1}},\left.\psi\right|_{\gamma_{3}}}^{(2)},
\end{aligned}
$$

are contractions for $\varphi, \psi \in C\left(\widetilde{T}_{h}\right)$ and $n, m \in \mathbb{N}^{*}$.
Let $F, G \in X_{\left.\varphi\right|_{\gamma_{2}},\left.\varphi\right|_{\gamma_{3}}}^{(1)}$. From (3) we have

$$
\begin{aligned}
\mid B_{m}^{x}(F) & (x, y)-B_{m}^{x}(G)(x, y) \mid \\
= & \left|B_{m}^{x}(F-G)(x, y)\right| \\
\leq & \left|1-\left(1-\frac{x-g_{2}(y)}{g_{3}(y)-g_{2}(y)}\right)^{m}-\left(\frac{x-g_{2}(y)}{g_{3}(y)-g_{2}(y)}\right)^{m}\right| \\
& \|F-G\|_{\infty} \leq\left(1-\frac{1}{2^{m-1}}\right)\|F-G\|_{\infty},
\end{aligned}
$$

where $\|\cdot\|_{\infty}$ denotes the Chebyshev norm. So,

$$
\begin{aligned}
& \left\|B_{m}^{x}(F)(x, y)-B_{m}^{x}(G)(x, y)\right\|_{\infty} \\
& \quad \leq\left(1-\frac{1}{2^{m-1}}\right)\|F-G\|_{\infty}, \quad \forall F, G \in X_{\left.\varphi\right|_{\gamma_{2}},\left.\varphi\right|_{\gamma_{3}} ^{(1)}},
\end{aligned}
$$

that is, $\left.B_{m}^{x}\right|_{X_{\left.\varphi\right|_{\gamma_{2}},\left.\varphi\right|_{\gamma_{3}}}^{(1)}}$ is a contraction for $\varphi \in C\left(\widetilde{T}_{h}\right)$.

Analogously we have

$$
\begin{aligned}
\mid B_{n}^{y}(F) & (x, y)-B_{n}^{y}(G)(x, y) \mid \\
= & \left|B_{n}^{y}(F-G)(x, y)\right| \\
\leq & \left|1-\left(1-\frac{y-f_{1}(x)}{f_{3}(x)-f_{1}(x)}\right)^{n}-\left(\frac{y-f_{1}(x)}{f_{3}(x)-f_{1}(x)}\right)^{n}\right| \\
& \|F-G\|_{\infty} \leq\left(1-\frac{1}{2^{n-1}}\right)\|F-G\|_{\infty},
\end{aligned}
$$

whence

$$
\begin{aligned}
& \left\|B_{n}^{y}(F)(x, y)-B_{n}^{y}(G)(x, y)\right\|_{\infty} \\
& \leq\left(1-\frac{1}{2^{n-1}}\right)\|F-G\|_{\infty}, \quad \forall F, G \in X_{\left.\psi\right|_{\gamma_{1}},\left.\psi\right|_{\gamma_{3}} ^{(2)}},
\end{aligned}
$$

that is, $\left.B_{n}^{y}\right|_{X_{\left.\psi\right|_{1},\left.\psi\right|_{\gamma_{3}}}^{(2)}}$ is a contraction for $\psi \in C\left(\widetilde{T}_{h}\right)$.

On the other hand, $\left(\left(\left.\varphi\right|_{\gamma_{3}}-\left.\varphi\right|_{\gamma_{2}}\right) /\left(g_{3}(y)-g_{2}(y)\right)\right)(\cdot)+$ $\left(\left.g_{3}(y) \varphi\right|_{\gamma_{2}}-\left.g_{2}(y) \varphi\right|_{\gamma_{3}}\right) /\left(g_{3}(y)-g_{2}(y)\right) \in X_{\left.\varphi\right|_{\gamma_{2}},\left.\varphi\right|_{\gamma_{3}}}^{(1)},\left(\left(\left.\psi\right|_{\gamma_{3}}-\right.\right.$ $\left.\left.\left.\psi\right|_{\gamma_{1}}\right) /\left(f_{3}(x)-f_{1}(x)\right)\right)(\cdot)+\left(\left.f_{3}(x) \psi\right|_{\gamma_{1}}-\left.f_{1}(x) \psi\right|_{\gamma_{3}}\right) /\left(f_{3}(x)-\right.$ $\left.f_{1}(x)\right) \in X_{\left.\psi\right|_{\gamma_{1}},\left.\psi\right|_{\gamma_{3}}}^{(2)}$ are fixed points of $B_{m}^{x}$ and $B_{n}^{y}$; that is,

$$
\begin{array}{r}
B_{m}^{x}\left(\frac{\left.\varphi\right|_{\gamma_{3}}-\left.\varphi\right|_{\gamma_{2}}}{g_{3}(y)-g_{2}(y)}(\cdot)+\frac{\left.g_{3}(y) \varphi\right|_{\gamma_{2}}-\left.g_{2}(y) \varphi\right|_{\gamma_{3}}}{g_{3}(y)-g_{2}(y)}\right) \\
=\frac{\left.\varphi\right|_{\gamma_{3}}-\left.\varphi\right|_{\gamma_{2}}}{g_{3}(y)-g_{2}(y)}(\cdot)+\frac{\left.g_{3}(y) \varphi\right|_{\gamma_{2}}-\left.g_{2}(y) \varphi\right|_{\gamma_{3}}}{g_{3}(y)-g_{2}(y)}, \\
B_{n}^{y}\left(\frac{\left.\psi\right|_{\gamma_{3}}-\left.\psi\right|_{\gamma_{1}}}{f_{3}(x)-f_{1}(x)}(\cdot)+\frac{\left.f_{3}(x) \psi\right|_{\gamma_{1}}-\left.f_{1}(x) \psi\right|_{\gamma_{3}}}{f_{3}(x)-f_{1}(x)}\right) \\
=\frac{\left.\psi\right|_{\gamma_{3}}-\left.\psi\right|_{\gamma_{1}}}{f_{3}(x)-f_{1}(x)}(\cdot)+\frac{\left.f_{3}(x) \psi\right|_{\gamma_{1}}-\left.f_{1}(x) \psi\right|_{\gamma_{3}}}{f_{3}(x)-f_{1}(x)} .
\end{array}
$$


From the contraction principle, $F_{\left.\varphi\right|_{\gamma_{2}},\left.\varphi\right|_{\gamma_{3}}}^{(1)}(x, y):=\left(\left(\left.\varphi\right|_{\gamma_{3}}-\right.\right.$ $\left.\left.\left.\varphi\right|_{\gamma_{2}}\right) /\left(g_{3}(y)-g_{2}(y)\right)\right) x+\left(\left.g_{3}(y) \varphi\right|_{\gamma_{2}}-\left.g_{2}(y) \varphi\right|_{\gamma_{3}}\right) /\left(g_{3}(y)-\right.$ $\left.g_{2}(y)\right)$ is the unique fixed point of $B_{m}^{x}$ in $X_{\left.\varphi\right|_{\gamma_{2}},\left.\varphi\right|_{\gamma_{3}}}^{(1)}$ and $\left.B_{m}^{x}\right|_{X_{\varphi \varphi_{\gamma_{2}}, \varphi \gamma_{\gamma_{3}}}^{(1)}}$ is a Picard operator, with

$$
\begin{aligned}
\left(B_{m}^{x, \infty} F\right)(x, y) & \\
= & \frac{F\left(g_{3}(y), y\right)-F\left(g_{2}(y), y\right)}{g_{3}(y)-g_{2}(y)} x \\
+ & \frac{g_{3}(y) F\left(g_{2}(y), y\right)-g_{2}(y) F\left(g_{3}(y), y\right)}{g_{3}(y)-g_{2}(y)},
\end{aligned}
$$

and, similarly, $F_{\left.\psi\right|_{\gamma_{1}},\left.\psi\right|_{\gamma_{3}}}^{(2)}(x, y):=\left(\left(\left.\psi\right|_{\gamma_{3}}-\left.\psi\right|_{\gamma_{1}}\right) /\left(f_{3}(x)-\right.\right.$ $\left.\left.f_{1}(x)\right)\right) y+\left(\left.f_{3}(x) \psi\right|_{\gamma_{1}}-\left.f_{1}(x) \psi\right|_{\gamma_{3}}\right) /\left(f_{3}(x)-f_{1}(x)\right)$ is the unique fixed point of $B_{n}^{y}$ in $X_{\left.\psi\right|_{\gamma_{1}}, \psi \psi_{\gamma_{3}}}^{(2)}$ and $\left.B_{n}^{y}\right|_{X_{\psi_{\gamma_{1}},\left.\psi\right|_{\gamma_{3}}}^{(2)}}$ is a Picard operator, with

$$
\begin{aligned}
\left(B_{n}^{y, \infty} F\right)(x, y)= & \frac{F\left(x, f_{3}(x)\right)-F\left(x, f_{1}(x)\right)}{f_{3}(x)-f_{1}(x)} y \\
& +\frac{f_{3}(x) F\left(x, f_{1}(x)\right)-f_{1}(x) F\left(x, f_{3}(x)\right)}{f_{3}(x)-f_{1}(x)} .
\end{aligned}
$$

Consequently, taking into account (ii), by Theorem 8 it follows that the operators $B_{m}^{x}$ and $B_{n}^{y}$ are weakly Picard operators.

Now we study the convergence of the product and Boolean sum operators (7) and (9).

Theorem 10. The operator $P_{m n}$ is a weakly Picard operator and

$$
\begin{aligned}
&\left(P_{m n}^{\infty} F\right)(x, y) \\
&=\frac{1}{\left[g_{3}(y)-g_{2}(y)\right]\left[f_{3}(x)-f_{1}(x)\right]} \\
& \times\left[g_{3}(y) f_{3}\left(x_{0}\right) F\left(x_{0}, f_{1}\left(x_{0}\right)\right)+g_{2}(y) f_{1}\left(x_{1}\right)\right. \\
& \quad \times F\left(x_{1}, f_{3}\left(x_{1}\right)\right)-g_{3}(y) f_{1}\left(x_{1}\right) \\
&\left.\quad \times F\left(x_{0}, f_{3}\left(x_{0}\right)\right)-g_{2}(y) f_{3}\left(x_{0}\right) F\left(x_{1}, f_{1}\left(x_{1}\right)\right)\right] \\
&+\frac{x}{\left[g_{3}(y)-g_{2}(y)\right]\left[f_{3}(x)-f_{1}(x)\right]} \\
& \times\left[f_{1}\left(x_{1}\right) F\left(x_{0}, f_{3}\left(x_{0}\right)\right)+f_{3}\left(x_{0}\right) F\left(x_{1}, f_{1}\left(x_{1}\right)\right)\right. \\
&-f_{3}\left(x_{0}\right) F\left(x_{0}, f_{1}\left(x_{0}\right)\right) \\
&\left.-f_{1}\left(x_{0}\right) F\left(x_{1}, f_{3}\left(x_{1}\right)\right)\right] \\
&+ \frac{y}{\left[g_{3}(y)-g_{2}(y)\right]\left[f_{3}(x)-f_{1}(x)\right]} \\
& \times {\left[g_{3}(y) F\left(x_{0}, f_{3}\left(x_{0}\right)\right)\right.}
\end{aligned}
$$

$$
\begin{aligned}
& \quad+g_{2}(y) F\left(x_{1}, f_{1}\left(x_{1}\right)\right)-g_{3}(y) \\
& \left.\quad \times F\left(x_{0}, f_{1}\left(x_{0}\right)\right)-g_{2}(y) F\left(x_{1}, f_{3}\left(x_{1}\right)\right)\right] \\
& +\frac{x y}{\left[g_{3}(y)-g_{2}(y)\right]\left[f_{3}(x)-f_{1}(x)\right]} \\
& \times\left[F\left(x_{0}, f_{1}\left(x_{0}\right)\right)+F\left(x_{1}, f_{3}\left(x_{1}\right)\right)\right. \\
& \left.\quad-F\left(x_{0}, f_{3}\left(x_{0}\right)\right)-F\left(x_{1}, f_{1}\left(x_{1}\right)\right)\right], \\
& \text { with } x_{0}=g_{2}(y), x_{1}=g_{3}(y) .
\end{aligned}
$$

Proof. Let $X_{\alpha, \beta, \gamma, \delta}=\left\{F \in C\left(\widetilde{T}_{h}\right) \mid F\left(x_{0}, f_{1}\left(x_{0}\right)\right)=\right.$ $\left.\alpha, F\left(x_{1}, f_{1}\left(x_{1}\right)\right) \stackrel{=}{=} \beta, F\left(x_{1}, f_{3}\left(x_{1}\right)\right)=\gamma, F\left(x_{0}, f_{3}\left(x_{0}\right)\right)=\delta\right\}$ and denote

$$
\begin{aligned}
& F_{\alpha, \beta, \gamma, \delta}(x, y) \\
&:=\left(g_{3}(y) f_{3}\left(x_{0}\right) \alpha+g_{2}(y) f_{1}\left(x_{1}\right) \gamma\right. \\
&\left.-g_{3}(y) f_{1}\left(x_{1}\right) \delta-g_{2}(y) f_{3}\left(x_{0}\right) \beta\right) \\
& \times\left(\left[g_{3}(y)-g_{2}(y)\right]\left[f_{3}(x)-f_{1}(x)\right]\right)^{-1} \\
&+ \frac{f_{1}\left(x_{1}\right) \delta+f_{3}\left(x_{0}\right) \beta-f_{3}\left(x_{0}\right) \alpha-f_{1}\left(x_{0}\right) \gamma}{\left[g_{3}(y)-g_{2}(y)\right]\left[f_{3}(x)-f_{1}(x)\right]} x \\
&+ \frac{g_{3}(y) \delta+g_{2}(y) \beta-g_{3}(y) \alpha-g_{2}(y) \gamma}{\left[g_{3}(y)-g_{2}(y)\right]\left[f_{3}(x)-f_{1}(x)\right]} y \\
&+ \frac{\alpha+\gamma-\beta-\delta}{\left[g_{3}(y)-g_{2}(y)\right]\left[f_{3}(x)-f_{1}(x)\right]} x y
\end{aligned}
$$

with $\alpha, \beta, \gamma, \delta \in \mathbb{R}$.

We remark that

(i) $X_{\alpha, \beta, \gamma, \delta}$ is a closed subset of $C\left(\widetilde{T}_{h}\right)$;

(ii) $X_{\alpha, \beta, \gamma, \delta}$ is an invariant subset of $P_{m n}$, for $\alpha, \beta, \gamma, \delta \in \mathbb{R}$ and $n, m \in \mathbb{N}^{*}$;

(iii) $C\left(\widetilde{T}_{h}\right)=\bigcup_{\alpha, \beta, \gamma, \delta} X_{\alpha, \beta, \gamma, \delta}$ is a partition of $C\left(\widetilde{T}_{h}\right)$;

(iv) $F_{\alpha, \beta, \gamma, \delta} \in X_{\alpha, \beta, \gamma, \delta} \cap F_{P_{m n}}$, where $F_{P_{m n}}$ denote the fixed points sets of $P_{m n}$.

The statements (i) and (iii) are obvious.

(ii), similarly with the proof of Theorem 9 , by linearity of Bernstein operators and Theorem 3, it follows that $X_{\alpha, \beta, \gamma, \delta}$ is an invariant subset of $P_{m n}$, for $\alpha, \beta, \gamma, \delta \in \mathbb{R}$ and $n, m \in \mathbb{N}^{*}$.

(iv), we prove that

$$
\left.P_{m n}\right|_{X_{\alpha, \beta, \gamma}, \delta}: X_{\alpha, \beta, \gamma, \delta} \longrightarrow X_{\alpha, \beta, \gamma, \delta}
$$

is a contraction for $\alpha, \beta, \gamma, \delta \in \mathbb{R}$ and $n, m \in \mathbb{N}^{*}$. Let $F, G \in$ $X_{\alpha, \beta, \gamma, \delta}$. From [7, Lemma 8] it follows that

$$
\begin{aligned}
& \left|P_{m n}(F)(x, y)-P_{m n}(G)(x, y)\right|=\left|P_{m n}(F-G)(x, y)\right| \\
& \quad \leq\left(1-\frac{1}{2^{m+n-2}}\right)\|F-G\|_{\infty} .
\end{aligned}
$$


So,

$$
\begin{aligned}
& \left\|P_{m n}(F)(x, y)-P_{m n}(G)(x, y)\right\|_{\infty} \\
& \quad \leq\left(1-\frac{1}{2^{m+n-2}}\right)\|F-G\|_{\infty}, \quad \forall F, G \in X_{\alpha, \beta, \gamma, \delta},
\end{aligned}
$$

that is, $\left.P_{m n}\right|_{X_{\alpha, \beta, \gamma, \delta}}$ is a contraction for $\alpha, \beta, \gamma, \delta \in \mathbb{R}$.

From the contraction principle we have that $F_{\alpha, \beta, \gamma, \delta}$ is the unique fixed point of $P_{m n}$ in $X_{\alpha, \beta, \gamma, \delta}$ and $\left.P_{m n}\right|_{X_{\alpha, \beta, \gamma, \delta}}$ is a Picard operator, so (26) holds. Consequently, taking into account (ii), by Theorem 8 it follows that the operator $P_{m n}$ is a weakly Picard operator.

Remark 11. We have a similar result for the operator $Q_{n m}$.

Theorem 12. The operator $S_{m n}$ is a weakly Picard operator and

$$
\begin{aligned}
&\left(S_{m n}^{\infty} F\right)(x, y) \\
&= \frac{F\left(g_{3}(y), y\right)-F\left(g_{2}(y), y\right)}{g_{3}(y)-g_{2}(y)} x \\
&+ \frac{g_{3}(y) F\left(g_{2}(y), y\right)-g_{2}(y) F\left(g_{3}(y), y\right)}{g_{3}(y)-g_{2}(y)} \\
&+ \frac{F\left(x, f_{3}(x)\right)-F\left(x, f_{1}(x)\right)}{f_{3}(x)-f_{1}(x)} y \\
&+ \frac{f_{3}(x) F\left(x, f_{1}(x)\right)-f_{1}(x) F\left(x, f_{3}(x)\right)}{f_{3}(x)-f_{1}(x)} \\
&- \frac{1}{\left[g_{3}(y)-g_{2}(y)\right]\left[f_{3}(x)-f_{1}(x)\right]} \\
& \times {\left[g_{3}(y) f_{3}\left(x_{0}\right) F\left(x_{0}, f_{1}\left(x_{0}\right)\right)+g_{2}(y) f_{1}\left(x_{1}\right)\right.} \\
& \times F\left(x_{1}, f_{3}\left(x_{1}\right)\right)-g_{3}(y) f_{1}\left(x_{1}\right) F\left(x_{0}, f_{3}\left(x_{0}\right)\right) \\
&\left.-g_{2}(y) f_{3}\left(x_{0}\right) F\left(x_{1}, f_{1}\left(x_{1}\right)\right)\right] \\
&- \frac{x}{\left[g_{3}(y)-g_{2}(y)\right]\left[f_{3}(x)-f_{1}(x)\right]} \\
& \times {\left[f_{1}\left(x_{1}\right) F\left(x_{0}, f_{3}\left(x_{0}\right)\right)+f_{3}\left(x_{0}\right) F\left(x_{1}, f_{1}\left(x_{1}\right)\right)\right.} \\
&\left.-f_{3}\left(x_{0}\right) F\left(x_{0}, f_{1}\left(x_{0}\right)\right)-f_{1}\left(x_{0}\right) F\left(x_{1}, f_{3}\left(x_{1}\right)\right)\right] \\
&- \frac{x y}{\left[g_{3}(y)-g_{2}(y)\right]\left[f_{3}(x)-f_{1}(x)\right]} \\
& \times {\left[g_{3}(y) F\left(x_{0}, f_{3}\left(x_{0}\right)\right)+g_{2}(y) F\left(x_{1}, f_{1}\left(x_{1}\right)\right)\right.} \\
&\left.-g_{3}(y) F\left(x_{0}, f_{1}\left(x_{0}\right)\right)-g_{2}(y) F\left(x_{1}, f_{3}\left(x_{1}\right)\right)\right] \\
& \times {\left[F\left(x_{0}, f_{1}\left(x_{0}\right)\right)+F\left(x_{1}, f_{3}\left(x_{1}\right)\right)\right.} \\
&\left.-F\left(x_{0}, f_{3}\left(x_{0}\right)\right)-F\left(x_{1}, f_{1}\left(x_{1}\right)\right)\right], \\
& g_{0}=g_{2}(y), x_{1}=g_{3}(y) . \\
&
\end{aligned}
$$

Proof. The proof follows the same steps as in the previous theorems but using the following inequality:

$$
\begin{aligned}
& \left\|S_{m n}(F)(x, y)-S_{m n}(G)(x, y)\right\|_{\infty} \\
& \quad \leq\left[1-\left(\frac{1}{2^{m-1}}+\frac{1}{2^{n-1}}-\frac{1}{2^{m+n-2}}\right)\right]\|F-G\|_{\infty},
\end{aligned}
$$

in order to prove that $S_{m n}$ is a contraction.

Remark 13. We have a similar result for the operator $T_{n m}$.

\section{Conflict of Interests}

The authors declare that there is no conflict of interests regarding the publication of this paper.

\section{References}

[1] P. Blaga, T. Cătinaş, and G. Coman, "Bernstein-type operators on a triangle with all curved sides," Applied Mathematics and Computation, vol. 218, no. 7, pp. 3072-3082, 2011.

[2] P. Blaga, T. Cătinaş, and G. Coman, "Bernstein-type operators on a triangle with one curved side," Mediterranean Journal of Mathematics, vol. 9, no. 4, pp. 833-845, 2012.

[3] P. Blaga, T. Cătinaş, and G. Coman, "Bernstein-type operators on a square with one and two curved sides," Studia Universitatis Babeş-Bolyai, vol. 55, no. 3, pp. 51-67, 2010.

[4] G. Coman and T. Cătinaş, "Interpolation operators on a triangle with one curved side," BIT Numerical Mathematics, vol. 50, no. 2, pp. 243-267, 2010.

[5] I. A. Rus, "Picard operators and applications," Scientiae Mathematicae Japonicae, vol. 58, no. 1, pp. 191-219, 2003.

[6] O. Agratini and I. A. Rus, "Iterates of a class of discrete linear operators via contraction principle," Commentationes Mathematicae Universitatis Carolinae, vol. 44, no. 3, pp. 555$563,2003$.

[7] O. Agratini and I. A. Rus, "Iterates of some bivariate approximation processvia weakly Picard operators," Nonlinear Analysis Forum, vol. 8, no. 2, pp. 159-168, 2003.

[8] T. Cătinaş and D. Otrocol, "Iterates of Bernstein type operators on a square with one curved side via contraction principle," Fixed Point Theory, vol. 14, no. 1, pp. 97-106, 2013.

[9] T. Cătinaş and D. Otrocol, "Iterates of multivariate CheneySharma operators," Journal of Computational Analysis and Applications, vol. 15, no. 7, pp. 1240-1246, 2013.

[10] I. A. Rus, "Iterates of Stancu operators, via contraction principle," Studia Universitatis Babeş-Bolyai, vol. 47, no. 4, pp. 101-104, 2002.

[11] I. A. Rus, "Iterates of Bernstein operators, via contraction principle," Journal of Mathematical Analysis and Applications, vol. 292, no. 1, pp. 259-261, 2004.

[12] I. A. Rus, "Fixed point and interpolation point set of a positive linear operator on $C(\bar{D})$," Studia Universitatis Babeş-Bolyai, vol. 55, no. 4, pp. 243-248, 2010.

[13] H. Gonska, D. Kacsó, and P. Piţul, "The degree of convergence of over-iterated positive linear operators," Journal of Applied Functional Analysis, vol. 1, no. 4, pp. 403-423, 2006.

[14] H. Gonska and I. Raşa, "The limiting semigroup of the Bernstein iterates: degree of convergence," Acta Mathematica Hungarica, vol. 111, no. 1-2, pp. 119-130, 2006. 
[15] H. Gonska, P. Piţul, and I. Raşa, "Over-iterates of BernsteinStancu operators," Calcolo, vol. 44, no. 2, pp. 117-125, 2007.

[16] I. Raşa, " $\mathrm{C}_{0}$-semigroups and iterates of positive linear operators: asymptotic behaviour," Rendiconti del Circolo Matematico di Palermo II, vol. 2, supplement 82, pp. 123-142, 2010.

[17] H. Gonska and I. Raşa, "On infinite products of positive linear operators reproducing linear functions," Positivity, vol. 17, no. 1, pp. 67-79, 2013.

[18] G. Gwóźdź-Łukawska and J. Jachymski, "IFS on a metric space with a graph structure and extensions of the Kelisky-Rivlin theorem," Journal of Mathematical Analysis and Applications, vol. 356, no. 2, pp. 453-463, 2009.

[19] I. Gavrea and M. Ivan, "On the iterates of positive linear operators preserving the affine functions," Journal of Mathematical Analysis and Applications, vol. 372, no. 2, pp. 366-368, 2010.

[20] I. Gavrea and M. Ivan, "The iterates of positive linear operators preserving constants," Applied Mathematics Letters, vol. 24, no. 12, pp. 2068-2071, 2011.

[21] I. Gavrea and M. Ivan, "On the iterates of positive linear operators," Journal of Approximation Theory, vol. 163, no. 9, pp. 1076-1079, 2011.

[22] S. Karlin and Z. Ziegler, "Iteration of positive approximation operators," Journal of Approximation Theory, vol. 3, pp. 310-339, 1970.

[23] R. P. Kelisky and T. J. Rivlin, "Iterates of Bernstein polynomials," Pacific Journal of Mathematics, vol. 21, pp. 511-520, 1967.

[24] M. Crăciun, "Approximation operators constructed by means of Sheffer sequences," Revue d'Analyse Numérique et de Théorie de l'Approximation, vol. 30, no. 2, pp. 135-150, 2001.

[25] M. Crăciun and A. di Bucchianico, "Sheffer sequences, probability distributions and approximation operators," SPOR Report 2005-04, Department of Mathematics and Computer Science, Technische Universiteit Eindhoven, 2005.

[26] D. D. Stancu and M. R. Occorsio, "On approximation by binomial operators of Tiberiu Popoviciu type," Revue d'Analyse Numérique et de Théorie de l'Approximation, vol. 27, no. 1, pp. 167-181, 1998. 


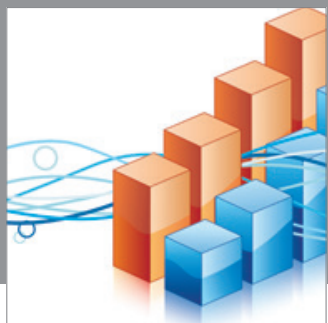

Advances in

Operations Research

mansans

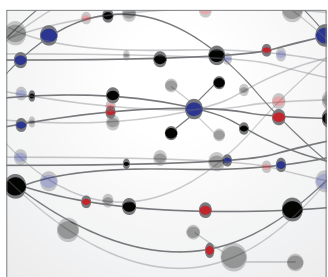

The Scientific World Journal
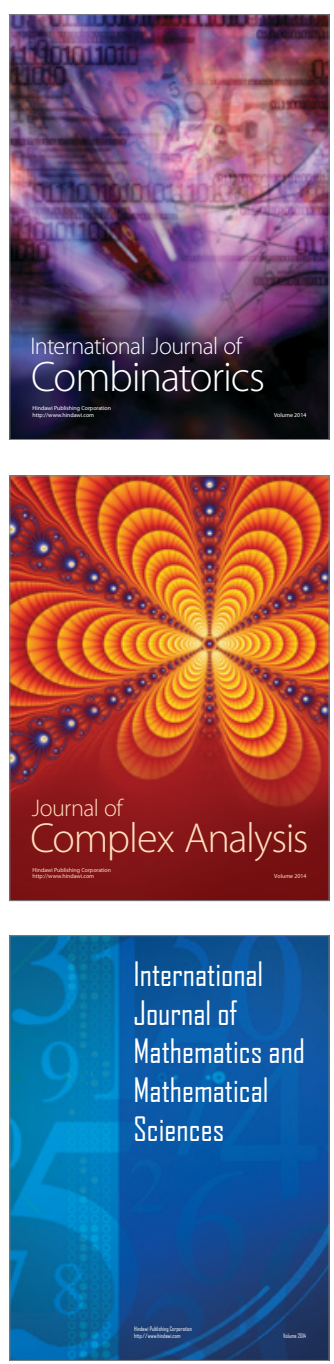
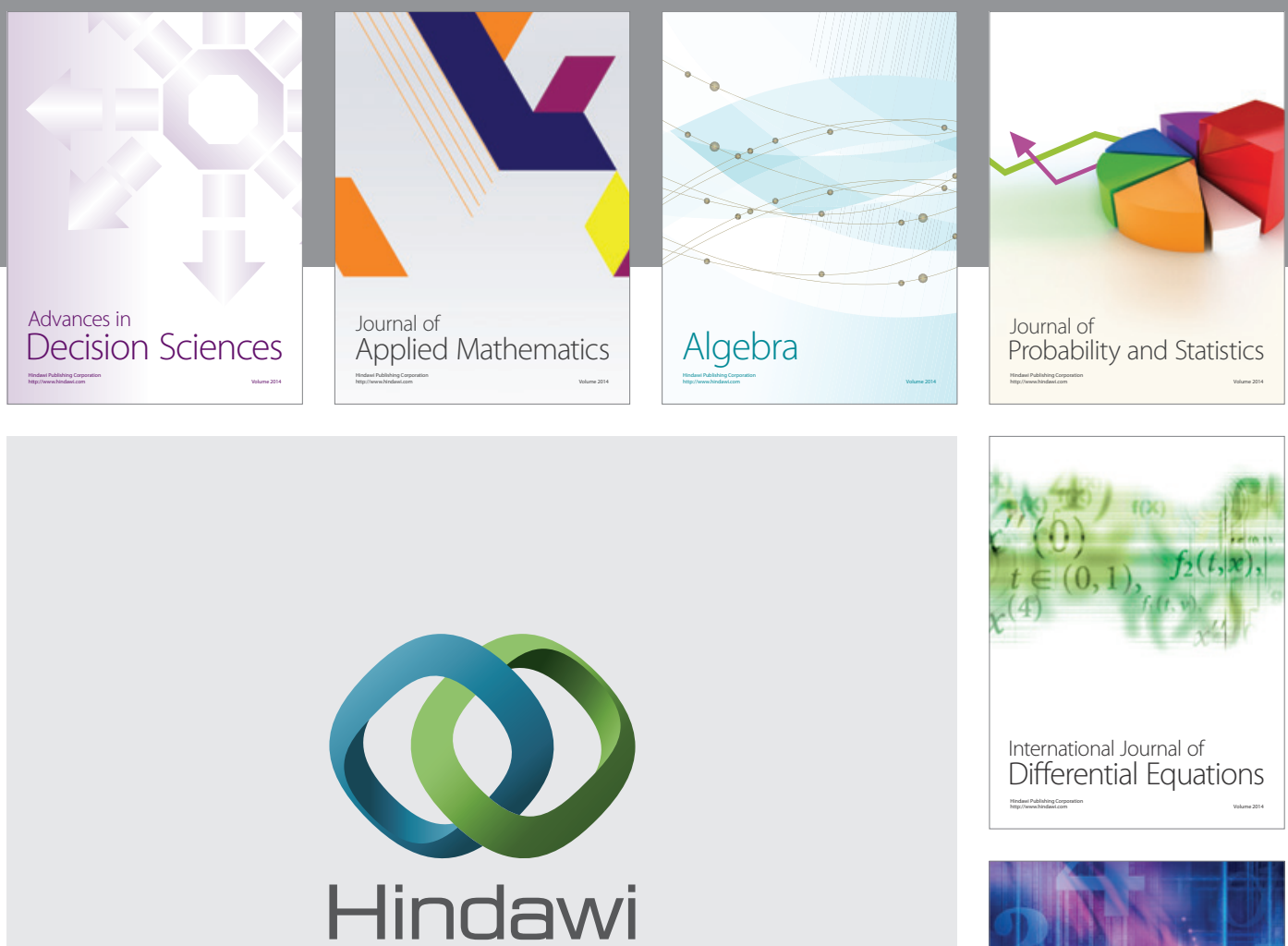

Submit your manuscripts at http://www.hindawi.com
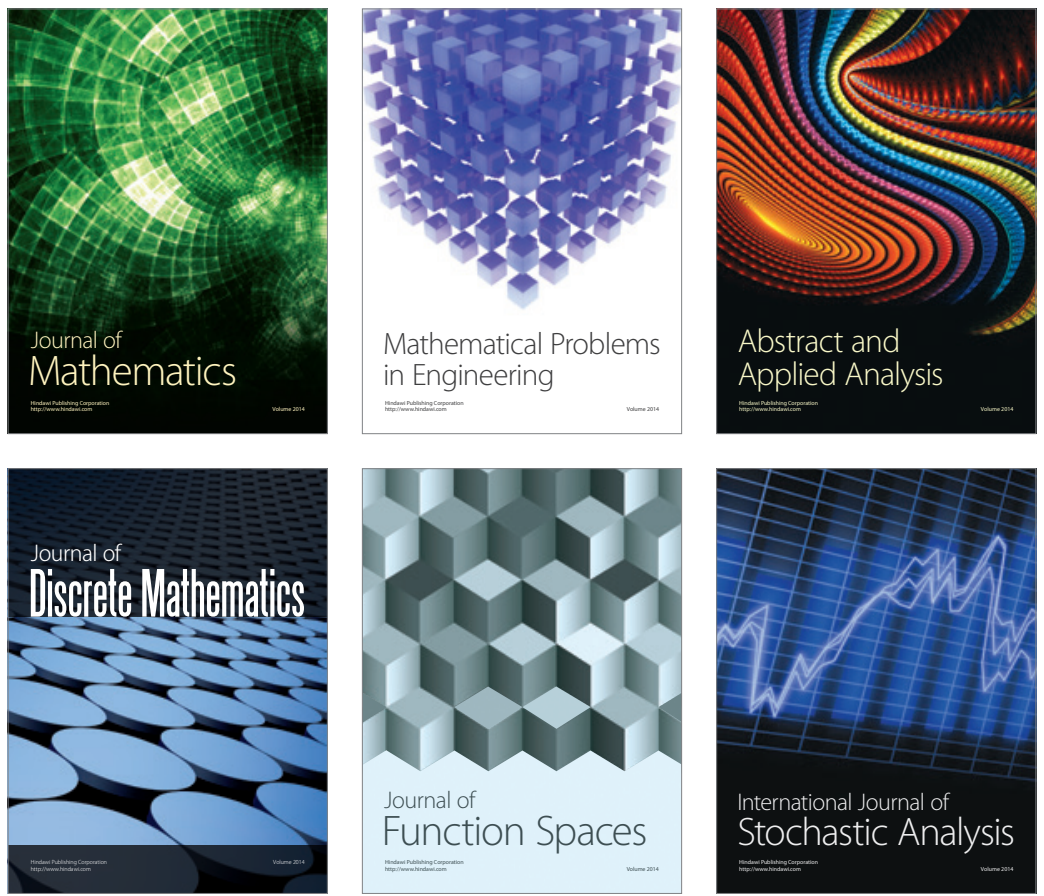

Journal of

Function Spaces

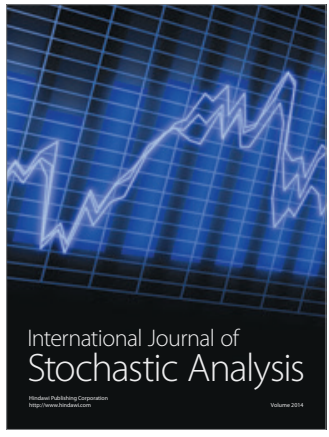

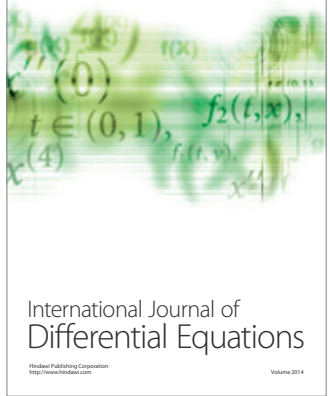
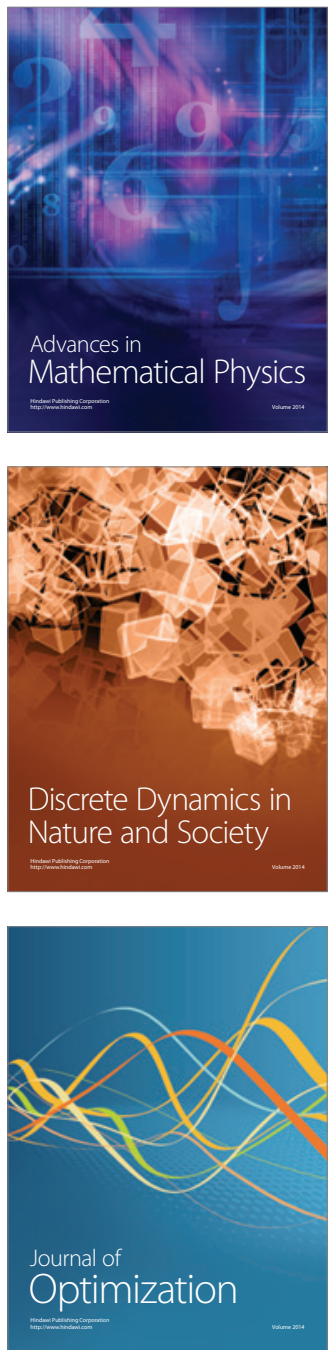